\title{
Water Purification using Recycled Polymeric Microfiltration Membranes.
}

\author{
Mostafa Khaled \\ Materials Engineering and Mechanical \\ Design \\ Faculty of Energy Engineering, Aswan \\ University \\ Aswan, Egypt \\ Mostafa_kh@aswu.edu.eg
}

\author{
A. H. El-Shazly \\ Chemical and Petrochemicals Engineering \\ Egypt-Japan University of Science and \\ Technology (E-JUST) \\ Alexandria, Egypt \\ Ahmed.elshazly@ejust.edu.eg
}

\author{
H. Noby \\ Materials Engineering and Mechanical \\ Design \\ Faculty of Energy Engineering, Aswan \\ University \\ Aswan, Egypt \\ Hussien379@energy.aswu.edu.eg
}

\author{
W. A. Aissa \\ Mechanical Engineering \\ Faculty of Energy Engineering, Aswan \\ University \\ Aswan, Egypt \\ Waessa@energy.aswu.edu.eg
}

\author{
Eman S. Mansor \\ Water Pollution Dep., Environmental \\ Research Division \\ National Research Centre, \\ Giza, Egypt \\ eman_mansor31@yahoo.com
}

\begin{abstract}
In our previous work, microfiltration membranes were successfully manufactured by non-solvent induced phase separation (NIPS) method using two concentrations (30 and 35 wt \%) of recycled high impact polystyrene (HIPS-R). N, Ndimethyl formamide (DMF) was used as solvent and water as a coagulation bath. These membranes were characterized in terms of chemical composition, surface hydrophilicity, surface crosssection morphology, porosity, and pores size distribution. Accordingly, the membrane's surfaces showed a semihydrophilic behavior with contact angles of $81^{\circ}$ and $91^{\circ}$ for 30 wt $\%$ and $35 \mathrm{wt} \%$ membranes respectively. In the current study, The prepared membranes were examined for the removal of Humic acid (HA) and Rhodamine $B$ (RhB) dye in a microfiltration process. Filtration experiment showed that pure water flux of $30 \mathrm{wt} \%$ membrane was higher than of $35 \mathrm{wt} \%$ membrane, also $30 \mathrm{wt} \%$ membrane has a higher humic acid and Dye removal efficiency than of $35 \mathrm{wt} \%$ membrane. Thus, the results suggest active membranes could be obtained using recycled high impact polystyrene. And then, solve the polymer waste accumulation problem in parallel with help in drinking water crisis solution.
\end{abstract}

Keywords-porous membranes, recycled waste, filtration, and hydrophilicity.

\section{INTRODUCTION}

The demand for pure water is a severe environmental concern [1]. Polymeric membranes are widely used recently for water purification. Wastewater treatment, modifying the membranes to give superior performance, like performing higher fluxes with higher rejection and lower fouling, all of which reduces energy usage, becomes a target [1-2]. In the water purification process, natural organic materials, such as dyes and acids, are removed by ultrafiltration (UF) or microfiltration (MF) [2]. Humic acid (HA) is usually considered as a microbial incubator in water pipes, membrane foulant, and primary source of carcinogenic and mutagenic compounds such as disinfection byproducts [16]. Besides, Rhodamine $\mathrm{B}(\mathrm{RhB})$ dye also has a harmful biological impact when drained into water. $\mathrm{RhB}$ dye existence in water may cause the death of aquatic species, photosynthetic activities. Complex in nature due to their aromatic structures, which are nonbiodegradable as a result of their optical, thermal and physic-chemical stability [17].

Polymeric membranes as polymer-based materials have distinctive characteristics, like lightweight, easy manufacturing, low cost, and even electrical conductivity as in conductive polymers [3]. Accordingly, their usage covers several fields and applications. Plastics are produced at a considerable rate may reach 230 million tons annually. Besides, plastics left behind a large amount of waste, about $12 \%$ of the solid waste. Efficient treatment of waste polymers is still a severe challenge. Combustion or burying underground are conventional methods to eliminate plastics waste amounts. Unfortunately, these methods have adverse effects on the environment. Consequently, to limit the side effects of polymer waste, plastic recycling is highly considered [4]. Among these polymers that can be recycled, high-impact polystyrene (HIPS) which is formed by the polybutadiene rubber and polystyrene (PS) interaction. HIPS showed significant advantages such as high impact resistance, stability, and ease preparation [1].

Polymers were used widely in separation applications [1,59]. There are different methods for the preparation of PS membranes due to their effective performance for many applications. Zhuang et al. fabricated flat sheet membranes from diverse types of waste PS for the gas separation [6]. It was found that the recycled high-impact polystyrene (HIPS-R) displayed excellent thermal stability and higher gas permeation performance [6]. Garcia et al. studied the application of HIPS$\mathrm{R}$ flat sheet membranes in low-pressure membrane processes [1]. An asymmetric structure with slightly higher porosity, when compared to the commercial HIPS membranes, were successfully obtained [1]. Bussi et al. fabricated PS membranes by phase inversion method with finger-like structure crosssectional [7]. Ke et al. used the electrospinning method to fabricate nanofibrous PS membranes with a superhydrophobic surface [8]. Microporous PS membranes with enhanced surface hydrophobicity were successfully synthesized in [9]. Furthermore, in our previous study [18], semihydrofilic HIPS- 
$\mathrm{R}$ membranes were successfully fabricated with different HIPS-R concentrations (20, 25, 30, $35 \mathrm{wt} \%)$.

In the current work, HIPS-R membranes with 30 and 35 $\mathrm{wt} \%$, were used in the microfiltration $(\mathrm{MF})$ process for water purification. HA and $\mathrm{RhB}$ dye were selected to be removed from their aqueous solution. The microfiltration process was investigated in terms of the permeate water flux and the rejection percentage.

\section{EXPERIMENTAL}

\section{A. Materials}

HIPS-R cups obtained from the local Egyptian market were used as the membrane's polymer base material. The cups were washed and dried before use, cut into small square pieces (5 $\mathrm{mm}$ average side length), ultrasonicated in distilled water, and dried overnight at $75^{\circ} \mathrm{C}$. N, N-dimethyl formamide (DMF) was employed as the organic solvent. Both DMF, HA, and Dye were purchased from Sigma-Aldrich. Distilled water was utilized for washing and solution preparation processes.

\section{B. HIPS-R membranes preparation}

The membranes preparation procedure is as mentioned in our previous study [18]. Typically, 30, and $35 \mathrm{wt} \%$ of HIPS-R were added to $10 \mathrm{~mL}$ DMF solvent with stirring at elevated temperature to prepare the polymer homogeneous solutions. Then the solutions were then cast on glass slides with a $200 \mu \mathrm{m}$ doctor blade at room temperature. Distilled water at room temperature was utilized as a coagulation bath. Finally the membranes were washed and dried overnight at $70^{\circ} \mathrm{C}$.

\section{Membrane characterization}

The surface and cross-sectional morphology of the prepared membranes were observed using a Field-emission scanning electron microscopy (FESEM, FEI Quanta 200 scanning electron microscope, FEI Company BV, Netherlands). Also, the pores' size and its distribution on the membranes' surface were analyzed using image analysis free software (Image-J). CA measurements (OCA 15EC Contact angle model, Data Physics Instrument Gmbh) were employed to measure the water contact angle of the prepared membranes. The average porosity of the surfaces of the membrane was calculated by the wet-dry weighting method. Accordingly, a small part of each membrane was soaked in water for $48 \mathrm{~h}$ until water filled the pores. Then, the excess water on the sample surface was removed. The surface porosity for each membrane was calculated using Eq. 1:

$$
\% \text { porosity }=\frac{\frac{W_{W}-W_{D}}{\rho_{W}}}{\frac{W_{W}-W_{D}}{\rho_{w}}+\frac{W_{D}}{\rho_{p}}} * 100
$$

where, $W w$ and $W_{D}$ are the weight of the wet and dry samples $(\mathrm{g})$, respectively. $\rho_{w}$ is the water density at room temperature $\left(\mathrm{g} / \mathrm{cm}^{3}\right)$, and $\rho_{p}$ is the density of the polymer $\left(\mathrm{g} / \mathrm{cm}^{3}\right)$.

\section{Filtration experiments}

Separation performance of the resulting membranes was studied in terms of permeate flux $(\mathrm{Jp}, \mathrm{L} / \mathrm{m} 2 \mathrm{~h})$ and rejection index $(\mathrm{R}, \%)$ by filtration experiments of $50 \mathrm{mg} / \mathrm{L}$ HA solutions and $15 \mathrm{mg} / \mathrm{L}$ Dye $(\mathrm{RhB})$ solutions. HA concentration was selected according to previous studies about membrane characterization [1]. These experiments were performed at 25 $\pm 1 \mathrm{oC}$ and 10 bars. HA and RhB rejections were measured by obtaining their concentrations in each stream using a UVVisible spectrophotometer (Hewlett-Packard 8453) at a wavelength of 254 and $543 \mathrm{~nm}$ for $\mathrm{HA}$ and $\mathrm{RhB}$, respectively. Figure 1 represents a schematic diagram for the microfiltration unit used. Therefore, rejection index was calculated as follows:

$$
\mathrm{R}(\%)=\frac{C_{f_{-}} C_{p}}{C_{f}} \cdot 100
$$

where, $C_{f}$ and $C_{p}$ are the solute concentration $(\mathrm{mg} / \mathrm{L})$ in the feed and permeate streams respectively.

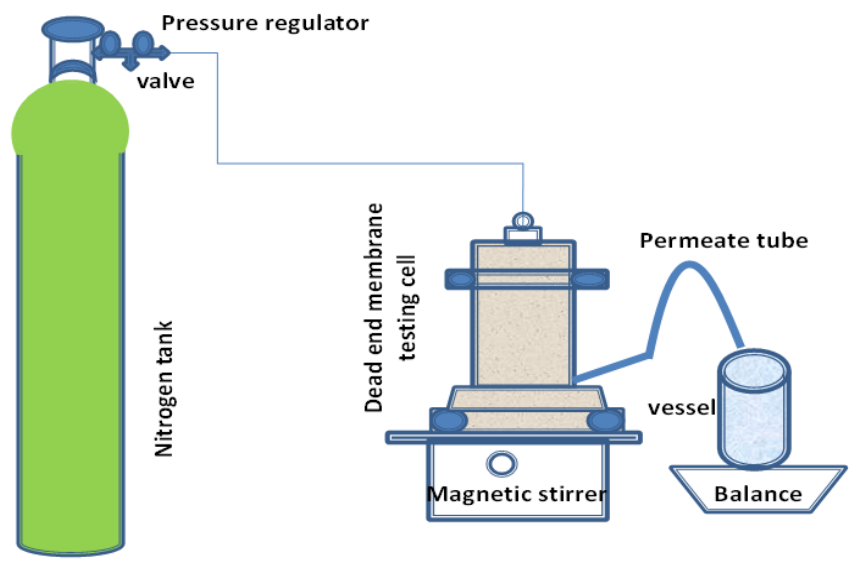

Fig.1. Schematic diagram of bench membrane testing cell.

\section{RESULTS AND DISCUSSION}

\section{A. Membranes properties}

As presented in our previous study [18], membranes with a sponge-like structure with noticeable longitudinal macrovoids were noticed, as shown in Fig. 2. These macrovoids are formed due to the high exchange rate between DMF (solvent) and distilled water (non-solvent) due to the high miscibility [1]. The walls around these voids have a sponge-like structure, especially for the $(35 \mathrm{wt} \%)$ polystyrene membrane. Furthermore, it was clearly illustrated that the surface membrane porosity reduced with the rise of polystyrene concentration.

Figure. 3 showed the pores diameter distribution and water contact angles of the produced membranes. The pore diameter distribution of $30 \mathrm{wt} \%$ membrane ranged from 0.1 to $1 \mu \mathrm{m}$ (see Figs. $3 \mathrm{a}$ and $3 \mathrm{~b}$ ), and the pore diameter distribution of $35 \mathrm{wt} \%$ membrane ranged from 0.1 to $0.5 \mu \mathrm{m}$. These results illustrated that the mean pore size decreased with the rise of HIPS-R concentration. Also, the membranes' surfaces showed a semihydrophilic behavior with contact angles of $83^{\circ}$ and $91^{\circ}$ for 30 wt $\%$ and $35 \mathrm{wt} \%$ membranes, respectively.The CA increased with the rise of the HIPS-R concentration.

Regarding the surface porosity It was recorded that the membrane with the PS concentration of $30 \mathrm{wt} \%$ displayed more significant surface porosity $(62.56 \%)$ than that of $35 \mathrm{wt} \%$ membrane $(61.6 \%)$. The porosity declined with the HIPS-R concentrations rise. The large size of the pores and the 
International Journal of Applied Energy Systems, Vol. 2, No. 2, July 2020 ISSN: 2636 - 3712 (Printed Version) ISSN: 2636 - 3720 (Online Version) Special Issue: ICEE-2019

formation of a large number of macrovoids in the case of 30 $\mathrm{wt} \%$ membrane may be the direct reason for the accomplished high porosity. With the increase of PS concentration, the pores size and numbers of macrovoids reduced which in turn lessened the overall porosity of the membrane.
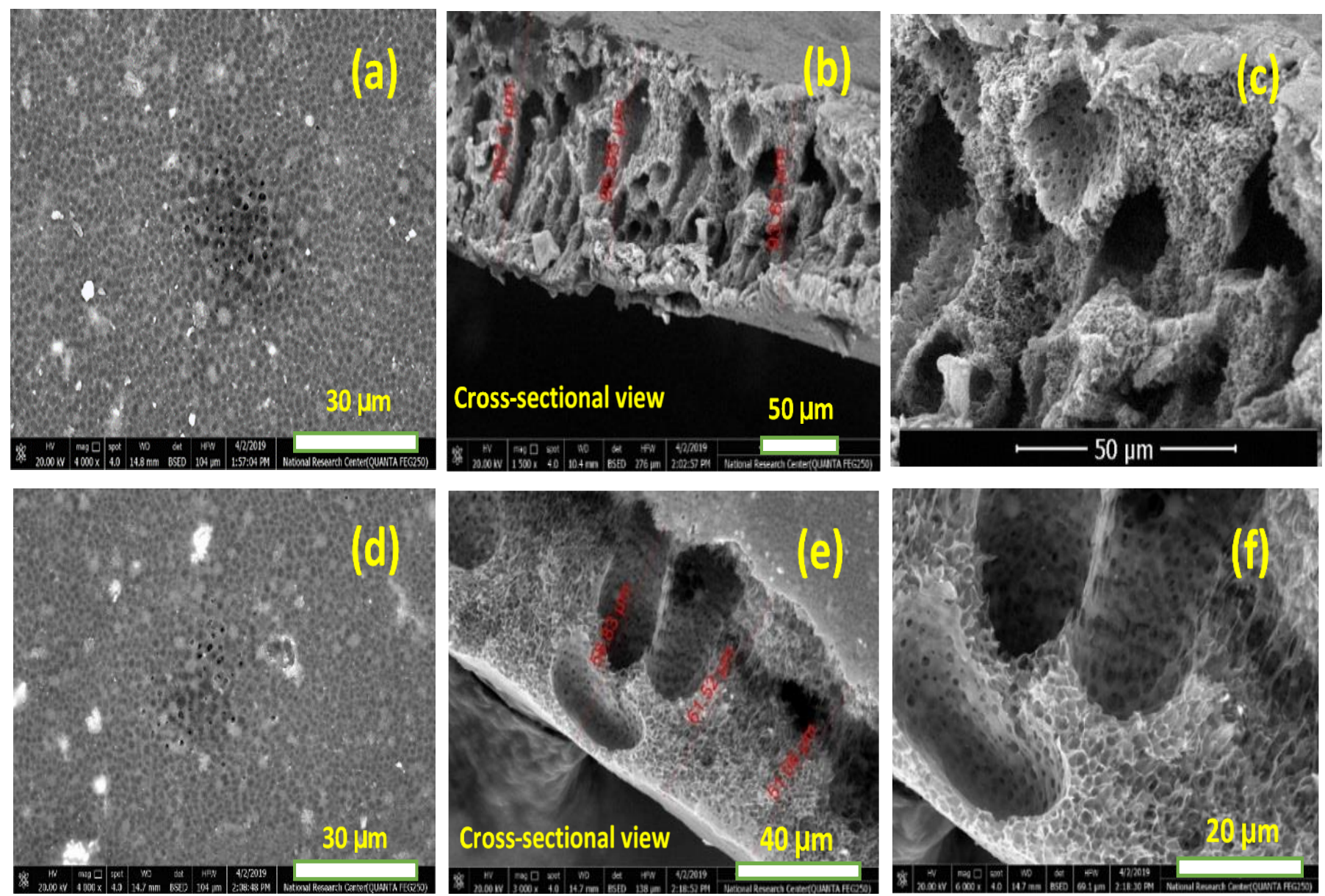

Fig. 2. SEM images of $30 \mathrm{wt} \%$ membrane (a) surface, (b) and (c) cross-sections, $35 \mathrm{wt} \%$ membrane (d) surface, (e) and (f) cross-sections.
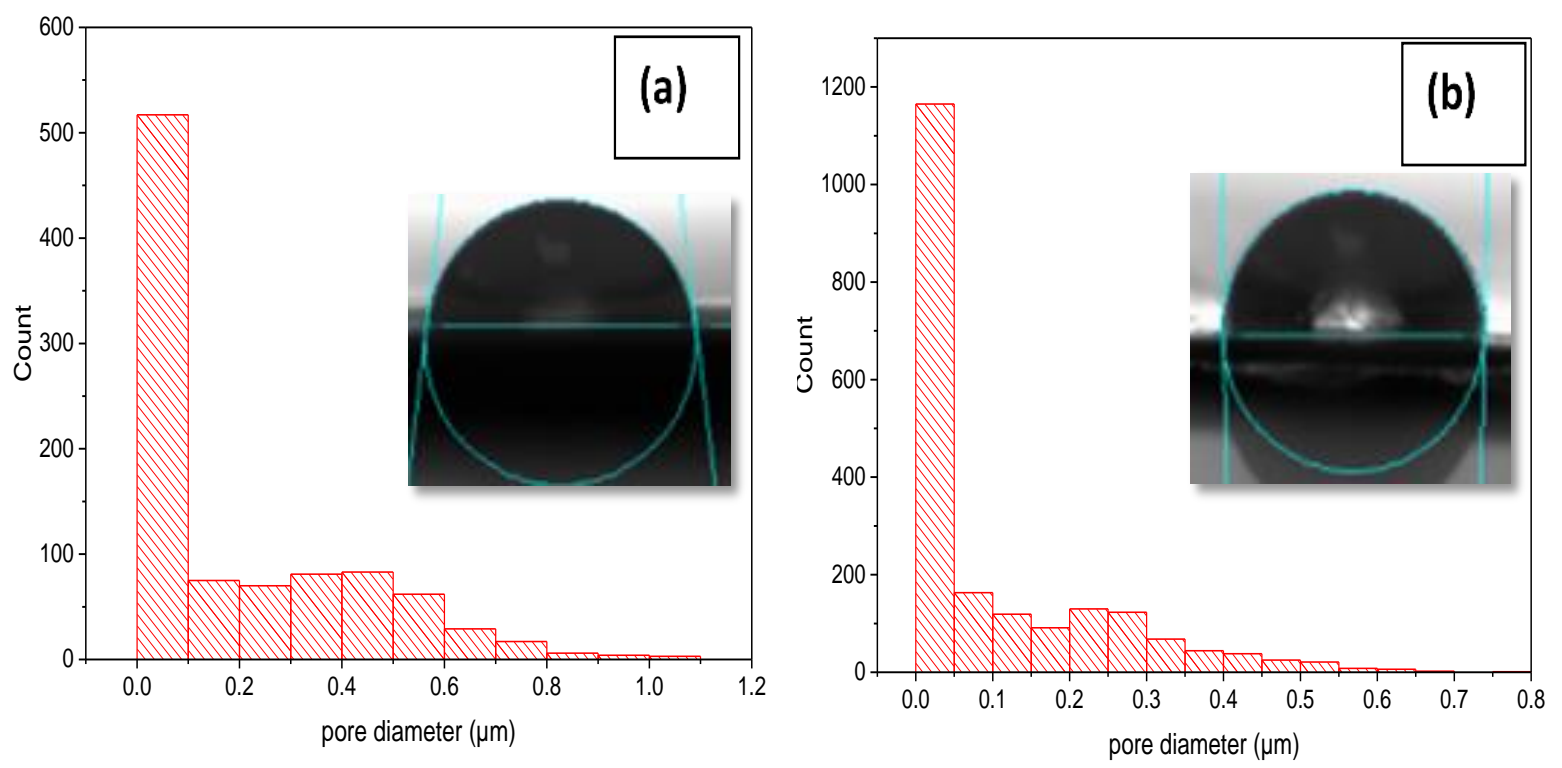

Fig. 3. Pore size distribution and water contact angle of (a) $30 \mathrm{wt} \%$, (b) $35 \mathrm{wt} \%$ membranes. 


\section{B. Filtration experiments}

Permeation flux and solute rejections are the main parameters to evaluate membrane performance [1]. Permeation flux values of $\mathrm{HA}, \mathrm{RhB}$, and water for both membranes are shown in Figs 4 and 5, The water permeability value of $30 \mathrm{wt} \%$ membrane was higher than that obtained for $35 \mathrm{wt} \%$ membrane, mainly due to the high porosity of $30 \mathrm{wt} \%$ membrane. The effect of the material and the porosity of membranes on their water flux was studied on many pieces of research [13]. The surface hydrophilicity of the membrane has a strong effect on the water flux thus high surface hydrophilicity leads to high water permeability. The $30 \mathrm{wt} \%$ membrane showed higher water permeability than $35 \mathrm{wt} \%$ membrane because it has higher surface hydrophilicity.

Finally, Fig. 9. shows the solute rejection of each membrane for HA and Dye solutions. The HA and Dye rejection index of 30 wt $\%$ membrane was higher than that obtained by $35 \mathrm{wt} \%$. Previous studies reported the large effect of porosity on water permeability [14]. The higher porosity and lower water contact angle could cause an improvement in hydrophilicity [15-16].

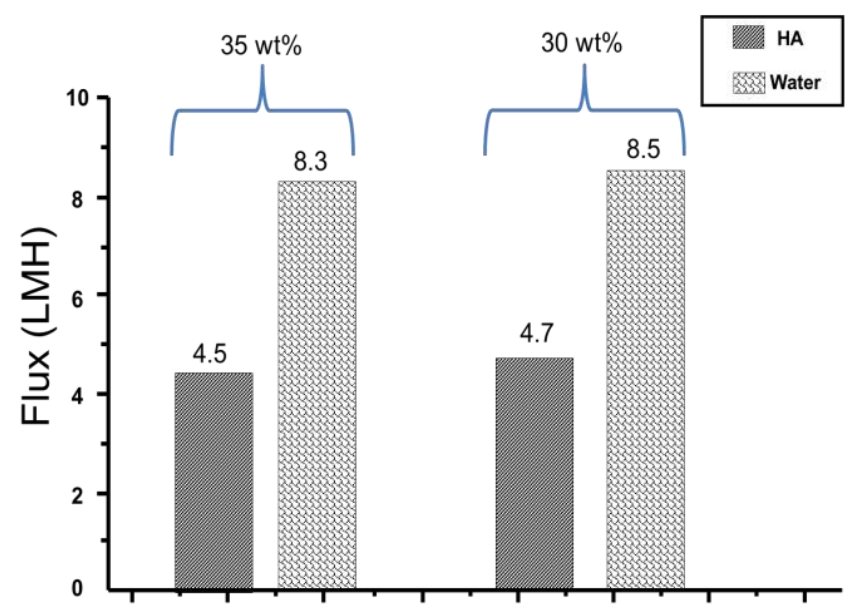

Fig. 4. The flux of the HA and water using both prepared membranes.

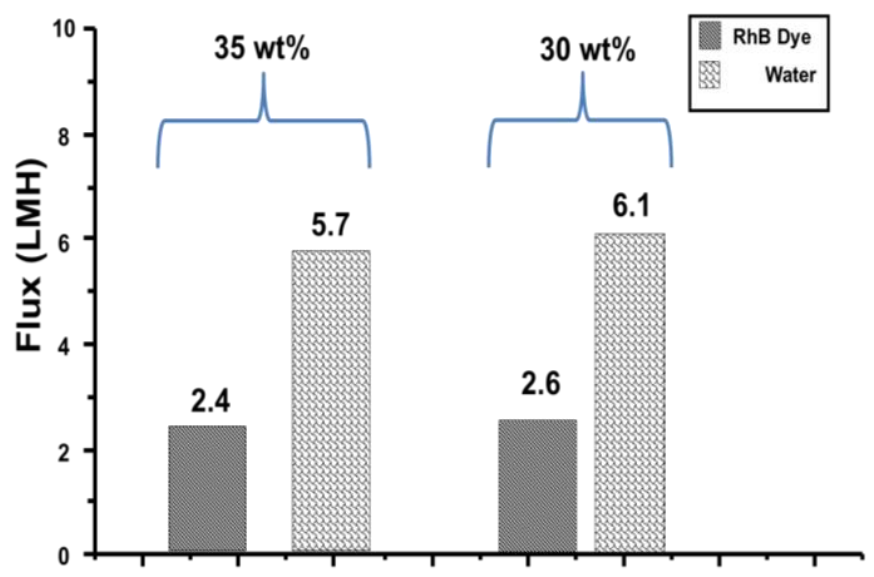

Fig. 5. The flux of the RhB dye and water using both prepared membranes.

The increasing in all these parameters (porosity, hydrophilicity, and water permeability) for $30 \mathrm{wt} \%$ membrane could be the reason for the improvement of their HA and Dye rejection index, especially the membrane hydrophilicity which inhibits interactions between the membrane surface and the organic solutes[14].

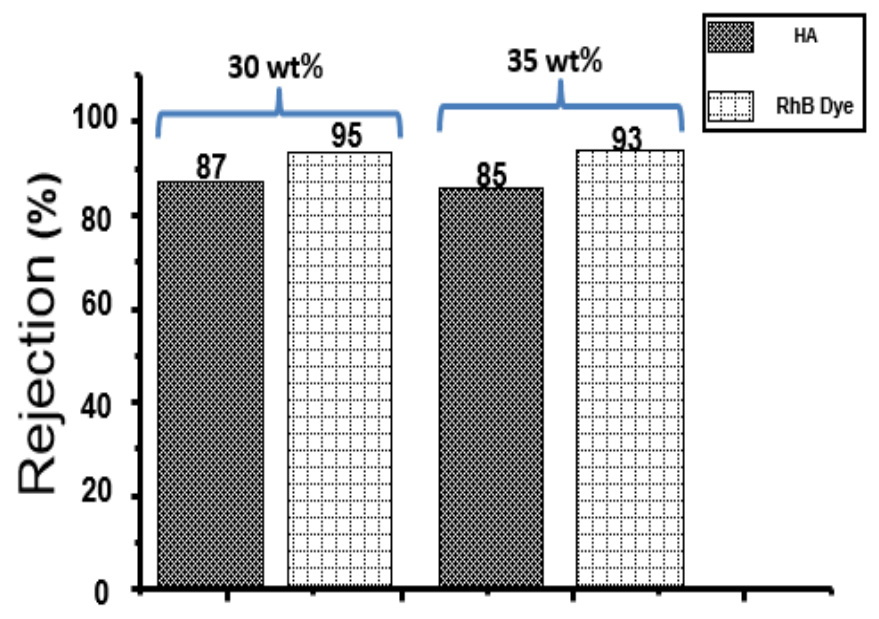

Fig. 6. Rejection of HA and RhB dye using both prepared membranes.

\section{CONCLUSION}

Tow hydrophilic flat sheet membranes were successfully fabricated from recycled high impact polystyrene HIPS-R (30 and $35 \mathrm{wt} \%$ ) in DMF solution. These membranes showed a porous cross-sectional structure with macrovoids. The porosity decreased with the rise of HIPS-R concentrations. The diffusivity of the DMF/HIPS-R solution with the coagulant, water, effect on the formation of the macrovoids and the sponge structure was approved. Also, the porosity gradually diminished from 62.56 to $61.6 \%$ when the concentration raised from 30 to $35 \mathrm{wt} \%$, respectively. Contact angle measurements showed that the membranes had a semi-hydrophilic surface. 30 $\mathrm{wt} \%$ membrane has the largest water permeability $\left(8.5 \mathrm{~L} / \mathrm{m}^{2} \mathrm{~h}\right)$ and rejection index () due to its high porosity and hydrophilicity. Finally, these results suggested that polystyrene wastes can be used to prepare effective flat sheet membranes.

\section{references}

[1] J. Garcia-ivars, X. Wang-xu, and M. Iborra-clar, "Application of postconsumer recycled high-impact polystyrene in the preparation of phaseinversion membranes for low-pressure membrane processes," Sep. Purif. Technol., vol. 175, pp. 340-351, 2017.

[2] A. Mehrparvar, A. Rahimpour, and M. Jahanshahi, "Journal of the Taiwan Institute of Chemical Engineers Modified ultrafiltration membranes for humic acid removal,” J. Taiwan Inst. Chem. Eng., 2013.

[3] H. Noby, M. F. Elkady, and M. Ohshima, "Novel Preparation of a Selfassembled HCl-doped Polyaniline Nanotubes using Compressed CO2assisted Polymerization," Polymer (Guildf)., 2018.

[4] Y. Zare, "Recent progress on preparation and properties of nanocomposites from recycled polymers: A review," Waste Manag., vol. 33, no. 3, pp. 598-604, 2013.

[5] H. Noby, A. El-shazly, M. Elkady, and M. Ohshima, “Adsorption Pro les of Acid Dye Using Synthesized Polyaniline Nanostructure with Di erent Morphologies," vol. 50, no. 3, pp. 170-177, 2017.

[6] G. L. Zhuang, H. H. Tseng, and M. Y. Wey, "Feasibility of using waste polystyrene as a membrane material for gas separation," Chem. Eng. Res. Des., vol. 111, no. C, pp. 204-217, 2016.

[7] Y. Bussi, S. Golan, C. G. Dosoretz, and M. S. Eisen, "Synthesis, characterization and performance of polystyrene/PMMA blend 
membranes for potential water treatment," Desalination, vol. 431, no. December 2017, pp. 35-46, 2018.

[8] H. Ke et al., "Electrospun polystyrene nanofibrous membranes for direct contact membrane distillation,” J. Memb. Sci., vol. 515, pp. 86-97, 2016.

[9] Y. Wang et al., "Enhanced performance of superhydrophobic polypropylene membrane with modified antifouling surface for high salinity water treatment," Sep. Purif. Technol., 2018.

[10]A. M. Bernardes, J. Z. Ferreira, and C. A. Ferreira, "High-impact polystyrene / polyaniline membranes for acid solution treatment by electrodialysis : Preparation, evaluation, and chemical calculation," vol. 320, pp. 52-61, 2008.

[11]K. Hu et al., "Synthesis ,Structure , and Properties of High-Impact Polystyrene / Octavinyl Polyhedral Oligomeric Silsesquioxane Nanocomposites,"pp. 1-7, 2014

[12] J. P. Matinlinna, S. Areva, L. V. J. Lassila, and P. K. Vallittu, "Characterization of siloxane films on titanium substrate derived from three aminosilanes," pp. 1314-1322, 2004.

[13]S. Mohsenpour, A. Safekordi, M. Tavakolmoghadam, F. Rekabdar, and M. Hemmati, “AC SC,” Polymer (Guildf)., 2016.
[14]J. Garcia-ivars and B. Van Der Bruggen, "Author's Accepted Manuscript,” J. Memb. Sci., 2015.

[15]J. Lin, R. Zhang, W. Ye, N. Jullok, A. Sotto, and B. Van Der Bruggen, "Journal of Colloid and Interface Science Nano-WS 2 embedded PES membrane with improved fouling and permselectivity," J. Colloid Interface Sci., vol. 396, pp. 120-128, 2013.

[16] H. Abdallah, Jamil, T., Shaban, A., et al. (2017). Influence of the polyacrylonitrile proportion on the fabricated UF blend membranes' performance for humic acid removal. Journal of Polymer Engineering, 38(2), pp. 129-136. Retrieved 30 Sep. 2019, from doi:10.1515/polyeng2017-0003.

[17] A. Oyekanmi, Ahmad A, Hossain K, Rafatullah M. "Adsorption of Rhodamine B dye from aqueous solution onto acid treated banana peel: Response surface methodology, kinetics and isotherm studies." PLoS One, 14(5), 2019.

[18] M. Khaled, H. Noby, A. El-Shazly, W. A. Aissa, Facile Fabrication of Porous and Hydrophilic Polystyrene Membranes using Recycled Waste, $2^{\text {nd }}$ International Conference of Chemical, Energy and Environmental Engineering, ICCEEE 2019, Alexandria, Egypt. 